\title{
Representações sociais da terapia antirretroviral para pessoas vivendo com HIV*
}

Social representations of antiretroviral therapy for people living with HIV

Las representaciones sociales de la terapia antirretroviral para las personas que viven con el VIH

\section{Shaiene Naiara Oliveira de Araújo ${ }^{\mathrm{I}}$, Vivian Corrêa Nascimento ${ }^{\mathrm{II}}$, Felipe Kaezer dos Santos ${ }^{\mathrm{III}}$, Sergio Corrêa Marques $^{\mathrm{IV}}$, Denize Cristina de Oliveirav ${ }^{\mathrm{V}}$}

Resumo: Objetivo: analisar a representação social da terapia antirretroviral para pessoas vivendo com HIV. Método: estudo qualitativo, com a utilização da teoria das representações sociais. A coleta de dados incluiu 50 participantes, sendo realizada de fevereiro a setembro de 2020, utilizando um questionário de caracterização sociodemográfica e um formulário para evocações livres. O cenário do estudo foi uma unidade de tratamento de HIV na região metropolitana do Rio de Janeiro. A análise dos dados foi realizada pelo software EVOC. Resultados: o núcleo central da representação da terapia antirretroviral reforça o controle do HIV e a imagem do medicamento como algo bom. Conclusão: a estrutura representacional sugere que o grupo tende à normalização da terapia antirretroviral, fazendo referência a um medicamento que controla o HIV. O estudo possibilitou reconhecer os diferentes conteúdos representacionais da terapia antirretroviral para o grupo estudado, além da variada complexidade entre as imagens identificadas.

Descritores: Antirretrovirais; Terapia antirretroviral de alta atividade; HIV; Enfermagem; Cooperação e adesão ao tratamento

\begin{abstract}
Objective: analyzing the social representation of antiretroviral therapy for people living with HIV. Method: a qualitative study using the Theory of Social Representations. Data collection included 50 participants, being performed from February to September 2020 using a sociodemographic characterization questionnaire and a form for free evocations. The study scenario was an HIV treatment unit in the metropolitan region of Rio de Janeiro. Data analysis was performed by the EVOC software. Results: the central nucleus of the representation of antiretroviral therapy reinforces HIV control and the image of the drug as something good. Conclusion: the representational structure suggests that the group tends to normalize antiretroviral therapy, referring to a drug that controls HIV. The study made it possible to recognize the different representational contents of antiretroviral therapy for the studied group, in addition to the varied complexity among the images identified.
\end{abstract}

\footnotetext{
${ }^{\text {I }}$ Enfermeira, Faculdade de Enfermagem UERJ, Rio de Janeiro, Brasil. shaienenoa@gmail.com, Orcid: https://orcid.org/0000-0001-7829-8589 II Enfermeira, Faculdade de Enfermagem UERJ, Rio de Janeiro, Brasil. viviancorrean25@gmail.com, Orcid: https://orcid.org/0000-0002-9961-3274 III Enfermeiro, Doutor e Professor adjunto da Faculdade de Enfermagem UERJ, Rio de Janeiro, Brasil. prof.felipek@gmail.com, Orcid: https://orcid.org/0000-0002-2430-467X

IV Enfermeiro, Doutor e Professor Adjunto da Faculdade de Enfermagem da UERJ. Rio de Janeiro, Brasil. scmarques2012@gmail.com, Orcid: https://orcid.org/0000-0003-2597-4875

V Enfermeira, Pós Doutora e Professora Titular da Área de Pesquisa da Faculdade de Enfermagem UERJ. Rio de Janeiro, Brasil. dcouerj@gmail.com, Orcid: https://orcid.org/0000-0002-0830-0935

* Extraído da monografia de conclusão de curso "Representações sociais da terapia antirretroviral”, Curso de graduação em enfermagem, Universidade do Estado do Rio de Janeiro, 2020.
} 
Descriptors: Anti-retroviral agents; Antiretroviral therapy, Highly active; HIV; Nursing; Treatment follow-up and compliance

Resumen: Objetivo: analizar la representación social de la terapia antirretroviral para las personas que viven con el VIH. Método: un estudio cualitativo utilizando la Teoría de las Representaciones Sociales. La recolección de datos incluyó a 50 participantes, realizándolos de febrero a septiembre de 2020 utilizando un cuestionario de caracterización sociodemográfica y un formulario para evocaciones libres. El escenario del estudio fue una unidad de tratamiento del VIH en la región metropolitana de Río de Janeiro. El análisis de los datos fue realizado por el software EVOC. Resultados: el núcleo central de la representación de la terapia antirretroviral refuerza el control del VIH y la imagen del fármaco como algo bueno. Conclusión: la estructura representacional sugiere que el grupo tiende a normalizar la terapia antirretroviral, refiriéndose a un fármaco que controla el VIH. El estudio ha permitido reconocer los diferentes contenidos representacionales de la terapia antirretroviral para el grupo estudiado, además de la variada complejidad entre las imágenes identificadas.

Descriptores: Antirretrovirales; Terapia antirretroviral altamente activa; VIH; Enfermería; Cumplimiento y adherencia al tratamiento

\section{Introdução}

A epidemia da infecção pelo vírus da imunodeficiência humana (HIV) é ainda hoje, uma questão de destaque e relevância para a saúde pública, pois estima-se que mais de 37 milhões de pessoas em todo o mundo vivam nesta condição. Ademais, calcula-se que 1,7 milhão de novas infecções foram identificadas no ano de $2019 .{ }^{1}$

Dez anos após o surgimento dos primeiros casos de HIV, em 1987, o medicamento zidovudina $(\mathrm{AZT})$, que anteriormente era usado para pacientes com câncer, foi indicado como o primeiro capaz de reduzir a multiplicação do HIV. Em seguida, com a criação do Sistema Único de Saúde (SUS) e com a pressão de ativistas, em 1991, o fornecimento do AZT passa a ser gratuito para as pessoas vivendo com HIV. ${ }^{2}$

Em 1992, a combinação entre AZT e Videx inaugura o que ficou conhecido como um “coquetel”. A terapia dupla era, então, a única opção de tratamento, a partir de um alto custo e de um grau de eficácia abaixo do desejado. No entanto, naquela circunstância, esta reduziu em $50 \%$ a mortalidade por Síndrome da Imunodeficiência Adquirida (AIDS) no Brasil, aumentando em $80 \%$ a eficácia do tratamento das infecções oportunistas por HIV. ${ }^{3}$

Após a XI Conferência Internacional de AIDS, realizada em 1996 em Vancouver, Canadá, os 
3 | Araújo SNO, Nascimento VC, Santos FK, Marques SC, Oliveira DC

resultados obtidos com três fármacos associados: a terapia tripla, formada por zidovudina, lamivudina e efavirenz, serviu de impulso para novas ações judiciais. Pouco tempo depois, ainda em 1996, esses novos medicamentos tornaram-se disponíveis no Brasil, por meio da Lei 9.313/96, que regulamenta o acesso gratuito aos antirretrovirais no SUS. ${ }^{4-5}$

Após mais de três décadas e de diversos avanços, a atual terapia antirretroviral (TARV) tem por objetivo diminuir consideravelmente a carga viral no organismo do indivíduo, reduzindo a mortalidade e a incidência de infecções oportunistas, gerando, por fim, melhoria na qualidade de vida. Por esse motivo, a TARV tem um grande impacto na vida das pessoas vivendo com HIV, quando utilizada de maneira correta e de acordo com a terapêutica proposta. ${ }^{6}$

O uso correto dos TARV relaciona-se com fatores importantes para a adesão ao tratamento, uma vez que alguns autores tratam esse uso como a forma prescrita pelo profissional de saúde, respeitando suas orientações. Sendo assim, para uma adesão satisfatória, é necessário envolver os usuários dessa terapia na escolha de seu esquema terapêutico e de sua posologia, uma vez que a adesão é compreendida como um processo colaborativo entre o profissional e indivíduo, facilitando a manutenção e o sucesso do tratamento. Além disso, para que a adesão à TARV seja adequada, $80 \%$ das doses prescritas devem ser tomadas, sob o risco de consequente falha terapêtica. ${ }^{7-8}$

Embora existam avanços relevantes na qualidade de vida das pessoas vivendo com HIV, ainda há desafios, principalmente à adequada adesão ao tratamento, pois o seguimento adequado da TARV continua a ser difícil, devido ao uso contínuo dos medicamentos. ${ }^{4}$ Além disso, após o diagnóstico da infecção pelo HIV, o indivíduo enfrenta dificuldades de convivência e de aceitação da doença, o que pode ocasionar conflitos emocionais que dificultam a continuidade da TARV. Esse processo de enfrentamento vivido pelo indivíduo é importante para que o mesmo sinta confiança nas mudanças necessárias ao seu estilo de vida diante da infecção pelo HIV, sendo necessário que a equipe profissional elabore estratégias para auxiliá-lo. ${ }^{9}$

Cabe reforçar que o acolhimento por parte da equipe de saúde e a oferta de serviços de saúde são fatores que impactam a adesão ao tratamento, de forma que se tornam fundamentais para o 
Representações sociais da terapia antirretroviral para pessoas vivendo com HIV | 4

seguimento da TARV. Alguns sujeitos sentem-se confortáveis em compartilhar suas dúvidas e inseguranças, uma estratégia para suplantar eventuais empecilhos para uma adesão satisfatória. Além disso, o apoio profissional pode colaborar para o retorno do paciente ao serviço, visto que o indivíduo se sente amparado quando é bem recebido. Essa atitude é importante, principalmente no início do tratamento em que a sobrecarga emocional é maior. ${ }^{10}$

O profissional de enfermagem deve ser capaz de prestar um cuidado humanizado e voltado para o indivíduo que possui o diagnóstico de HIV positivo, para além das questões fisiológicas envolvidas, valorizando outras dimensões da existência humana. Por meio de consultas de enfermagem, é possível a construção do conhecimento de forma partilhada, a fim de promover o estímulo à adesão à terapia, conhecimento sobre o antirretroviral e mudanças no estilo de vida. ${ }^{10-11}$

As representações sociais acerca do HIV envolvem as fragilidades enfrentadas, além de produzirem imagens negativas, mesmo com o avanço do tratamento. Entretanto, relatam o a percepção positiva sobre à TARV relacionado à cronicidade da doença. ${ }^{12} \mathrm{~A}$ teoria das representações sociais se adequa a esta discussão por propiciar a elaboração do conhecimento social por meio das interações humanas, permitindo a construção de uma dada realidade. ${ }^{13}$ Permite, portanto, conhecer a realidade do grupo estudado, com consequente definição de ações específicas de cuidado. A partir do conhecimento comum a um dado grupo social, igualmente designado como senso comum, esta teoria proporciona a construção de uma dada realidade, ${ }^{14}$ tornando apropriada a utilização deste referencial. Portanto, ao utilizar a Teoria de Representações Sociais, pretende-se a identificação, a análise e a discussão dessas representações como elemento mediador da relação entre as pessoas vivendo com HIV e a TARV. Tendo observado a importância da terapia antirretroviral, definiu-se o objetivo de analisar a representação social da terapia antirretroviral para pessoas vivendo com HIV.

\section{Método}

Trata-se de uma pesquisa qualitativa, com a utilização da Teoria das Representações Sociais em sua abordagem estrutural. Este referencial é considerado como uma forma de entender as 
5 | Araújo SNO, Nascimento VC, Santos FK, Marques SC, Oliveira DC

impressões de grupos sobre objetos sociais definidos, uma vez que permite a imersão no cotidiano dos sujeitos. Essa teoria possibilita a compreensão acerca do conhecimento constituído por um grupo social, pois reconhece que há diferentes maneiras de se conhecer e de se comunicar, reguladas por diferentes objetivos. ${ }^{15}$

A abordagem estrutural dá ênfase especial à estruturação dos conteúdos cognitivos, ocupando-se de sua transformação a partir das práticas sociais. Está mais centrada, portanto, no aspecto constituído do que sobre o aspecto constituinte das representações e valoriza uma orientação metodológica experimental. Além de permitir estudar o conteúdo representacional a partir das conexidades dos elementos. ${ }^{16}$

O cenário de estudo foi o Serviço de Atendimento Especializado (SAE) de um centro municipal de saúde localizado na Baixada Fluminense, Rio de Janeiro. Trata-se de referência para o cuidado de pessoas que vivem com HIV no município de Duque de Caxias e cidades circunvizinhas. Sendo de caráter ambulatorial, este serviço é composto por consultórios para atendimento individual e sigiloso, onde atua a equipe multiprofissional, incluindo médicos, enfermeiros, psicólogos e assistentes sociais. Os participantes do estudo foram 50 indivíduos, de ambos os sexos, em uso da TARV e em acompanhamento regular na referida unidade, que, convidados, aceitaram contribuir com o estudo. Os critérios de inclusão foram: pessoas com sorologia para HIV em tratamento com a terapia antirretroviral na referida unidade; com 18 anos ou mais. Foram excluídos do estudo os indivíduos incapazes de responder às solicitações de forma independente.

O convite para a pesquisa aconteceu ao final do atendimento de enfermagem, sendo explicitados os seus objetivos. Em comum, integrantes do grupo estudado possuem o fato de viverem com HIV e manterem acompanhamento pela equipe de saúde em uma mesma unidade. No total, 14 indivíduos não foram incluídos, em função da incapacidade de produzir as informações solicitadas. Em função disso não integraram o grupo que compreendeu o corpus de análise, tendo sua participação encerrada ao final da coleta. Nenhum dos usuários do serviço se negou a participar ou desistiu de fazê-lo durante a coleta dos dados. 
Representações sociais da terapia antirretroviral para pessoas vivendo com HIV | 6

A coleta de dados foi realizada no período de fevereiro a setembro de 2020, com a utilização de um questionário para a caracterização sociodemográfica do grupo e de um formulário para o registro de evocações livres, obtidas mediante a utilização do termo indutor “antirretroviral”.

Os dados foram coletados pelos autores do estudo, que atuam no local da pesquisa por meio de um projeto de extensão universitária. Dessa forma, possuem proximidade dos participantes. Previamente à fase de coleta de dados, os pesquisadores se reuniram para um treinamento sobre os cuidados a serem observados para tanto. Foi solicitado aos participantes que proferissem cinco palavras após a verbalização do termo indutor pelas pesquisadoras, procedendo ao registro dos termos evocados em um formulário, observando a sequência em que eram enunciados. A coleta foi realizada de forma individual, em uma sala privativa.

Para a análise dos dados, os termos coletados foram organizados em uma planilha e padronizados em um dicionário de evocações. Na sequência, o corpus foi submetido à análise pelo software EVOC, versão 2015, que permite organizar as palavras de acordo com a frequência e a ordem de evocação. A partir da utilização EVOC, obteve-se um quadro de quatro casas, com os termos enunciadas pelos sujeitos distribuídas pelos quatro quadrantes.

Diante dos termos que integram o quadro de quatro casas, foi realizada a análise de similitude, que consiste da criação de uma estrutura gráfica para o mapeamento cognitivo. Foi possível identificar a conexão entre as palavras evocadas e construir a estrutura da representação, donde constam as forças de ligação, ampliando a compreensão acerca do objeto do estudo. ${ }^{17}$

Foram seguidas todas as recomendações e condutas éticas, em atenção à resolução 466, de 12 de dezembro de 2012, assim como as especificidades éticas da resolução 580, de 22 de março de 2018. A participação no estudo se deu de forma voluntária e com a assinatura do termo de consentimento livre e esclarecido. Após apreciação pelo COEP/UERJ, o projeto desta pesquisa foi aprovado por meio do parecer de número 3.807.397, de 23 de janeiro de 2020.

\section{Resultados}


7 | Araújo SNO, Nascimento VC, Santos FK, Marques SC, Oliveira DC

No total, foram incluídas 50 pessoas vivendo com HIV, 30\% compreendidas na faixa etária entre 31 e 40 anos; com relação ao sexo, $52 \%$ era do sexo masculino e $48 \%$ do sexo feminino; quanto à religião, 48\% declarou-se evangélica. Quanto à orientação sexual, 64\% se declarou heterossexual; houve predomínio de pessoas vivendo sozinhas (64\%) e indivíduos que trabalham (64\%). Quanto ao tempo de tratamento, $48 \%$ pacientes indicou período superior a 5 anos. No que se refere à terapia antirretroviral em uso no momento da coleta de dados, destacou-se a combinação de tenofovir, lamivudina e efavirenz, para $36 \%$ dos participantes.

Durante o processo de registro e organização das evocações, houve saturação dos dados, como se pode observar, a seguir, pela redução dos termos evocados após a padronização: a partir da utilização do termo indutor “antirretroviral”, foram obtidas 222 evocações, que após padronização resultaram em 38 palavras ou expressões, constituindo o corpus de análise.

Para a análise prototípica e, portanto, a obtenção do quadro de quatro casas foi utilizado o software $\operatorname{Evoc}^{\circledR}$, com definição das frequências mínima, média, ordem de evocação e rang médio de evocação. A frequência mínima correspondente à quantidade de vezes que um termo foi evocado foi de 9; a frequência média foi calculada em 12 e a ordem média de evocação (OME) que indica a ordem sequencial com que os termos foram evocados pelos participantes do grupo estudado foi calculada pelo software em 3,0. A partir desses parâmetros, o software indicou a distribuição das 11 palavras ou expressões obtidas nos quatro quadrantes. O Quadro 1 apresenta os conteúdos representacionais ordenados segundo os critérios de frequência e de OME.

Quadro 1 - Quadro de quatro casas referente às evocações ao termo indutor antirretroviral para pessoas vivendo com HIV. Rio de Janeiro, 2020 ( $\mathrm{n}=50$ sujeitos, Fmin= 9; Fmed=12; Rang médio=3,0) 
Representações sociais da terapia antirretroviral para pessoas vivendo com HIV $\mid 8$

\begin{tabular}{|c|c|c|c|c|c|c|}
\hline $\mathrm{OME}^{* *}$ & \multicolumn{3}{|c|}{$\operatorname{Rang}^{\star \star *}<3,00$} & \multicolumn{3}{|c|}{ Rang $\geq 3,00$} \\
\hline Fmed $^{*}$ & Evocações & Freq & $\mathrm{OME}^{\star *}$ & Evocações & Freq & OME \\
\hline \multirow{2}{*}{$\geq 12$} & NUCLEO CENTRAL & \multirow{2}{*}{$\begin{array}{l}26 \\
14 \\
12\end{array}$} & \multirow{2}{*}{$\begin{array}{l}2,538 \\
3,000 \\
1,417\end{array}$} & PRIMEIRA PERIFERIA & \multirow[t]{2}{*}{21} & \multirow[t]{2}{*}{3,952} \\
\hline & $\begin{array}{l}\text { Controla-HIV } \\
\text { Bom } \\
\text { Medicamento }\end{array}$ & & & Vida & & \\
\hline \multirow{2}{*}{$<11$} & ZONA DE CONTRASTE & \multirow{2}{*}{$\begin{array}{l}11 \\
9 \\
9 \\
9\end{array}$} & \multirow{2}{*}{$\begin{array}{l}2,636 \\
2,556 \\
2,667 \\
2,778\end{array}$} & SEGUNDA PERIFERIA & \multirow{2}{*}{$\begin{array}{l}11 \\
10 \\
10\end{array}$} & \multirow{2}{*}{$\begin{array}{l}3,273 \\
3,800 \\
3,200\end{array}$} \\
\hline & $\begin{array}{c}\text { Proteção } \\
\text { Diário } \\
\text { Ruim } \\
\text { Salvação }\end{array}$ & & & $\begin{array}{c}\text { Normal } \\
\text { Dependência } \\
\text { Tranquilidade }\end{array}$ & & \\
\hline
\end{tabular}

${ }^{\star}$ Fmed - Frequência Média; ${ }^{* \star}$ OME - ordem média de evocação; ${ }^{* \star *}$ Rang - media geral das ordens de evocação.

O quadrante superior esquerdo concentra termos associados ao núcleo central da representação, sendo estes evocados em maior quantidade e nas primeiras posições. Este quadrante indica os possíveis conteúdos centrais da representação da terapia antirretroviral para o grupo estudado. Nele encontram-se os termos controla-HIV, bom e medicamento. O termo "controlaHIV” está relacionado ao conhecimento do grupo a respeito da finalidade da TARV e apresenta a maior frequência da análise (26). Além disso, identifica-se um elemento avaliativo que qualifica a TARV expresso pelo termo "bom” (14), e finalizando observa-se um elemento imagético da TARV em "medicamento" (12) e que foi o termo mais prontamente evocado.

O quadrante superior direito (primeira periferia) apresenta a palavra que foi evocada também com maior frequência, porém mais tardiamente. É composto pelo termo “vida” (21), compreendido como uma evocação de cunho positivo que enaltece a sobrevivência em contraposição à morte associada à doença nos períodos iniciais da epidemia. O quadrante inferior direito (segunda periferia), é constituído pelos termos que foram evocados com menor frequência e de modo tardio. Neste quadrante, estão incluídos os termos “dependência” (10), "normal” (11) e "tranquilidade" (10). Nestas três evocações, observam-se, respectivamente, um elemento negativo referindo a relação de 
9 | Araújo SNO, Nascimento VC, Santos FK, Marques SC, Oliveira DC

dependência estabelecida entre o sujeito e o medicamento, um elemento que remete a tentativa de naturalização da TARV e um elemento de cunho positivo que remete ao enfrentamento da doença por meio da medicação resultando em tranquilidade.

O quadrante inferior esquerdo (zona de contraste) é formado pelas palavras que foram evocadas em menor quantidade, contudo, mais prontamente lembradas pelos participantes do estudo. Neste quadrante encontram-se os termos “proteção” (11), “diário” (9), "ruim” (9) e “salvação” (9). As imagens da proteção e da salvação representam uma atitude positiva em relação a TARV, ao passo que os termos "diário" e "ruim", atitudes negativas associadas à dependência estabelecida entre o sujeito e o medicamento. A conotação negativa observada nesse quadrante aponta para uma possível variação do núcleo central, podendo indicar um subgrupo que assume posicionamento menos positivo frente à TARV.

A partir da elaboração do quadro de quatro de casas, foi realizada a análise de similitude, baseada na força de ligação entre os termos evocados para o termo antirretroviral (Figura 1).

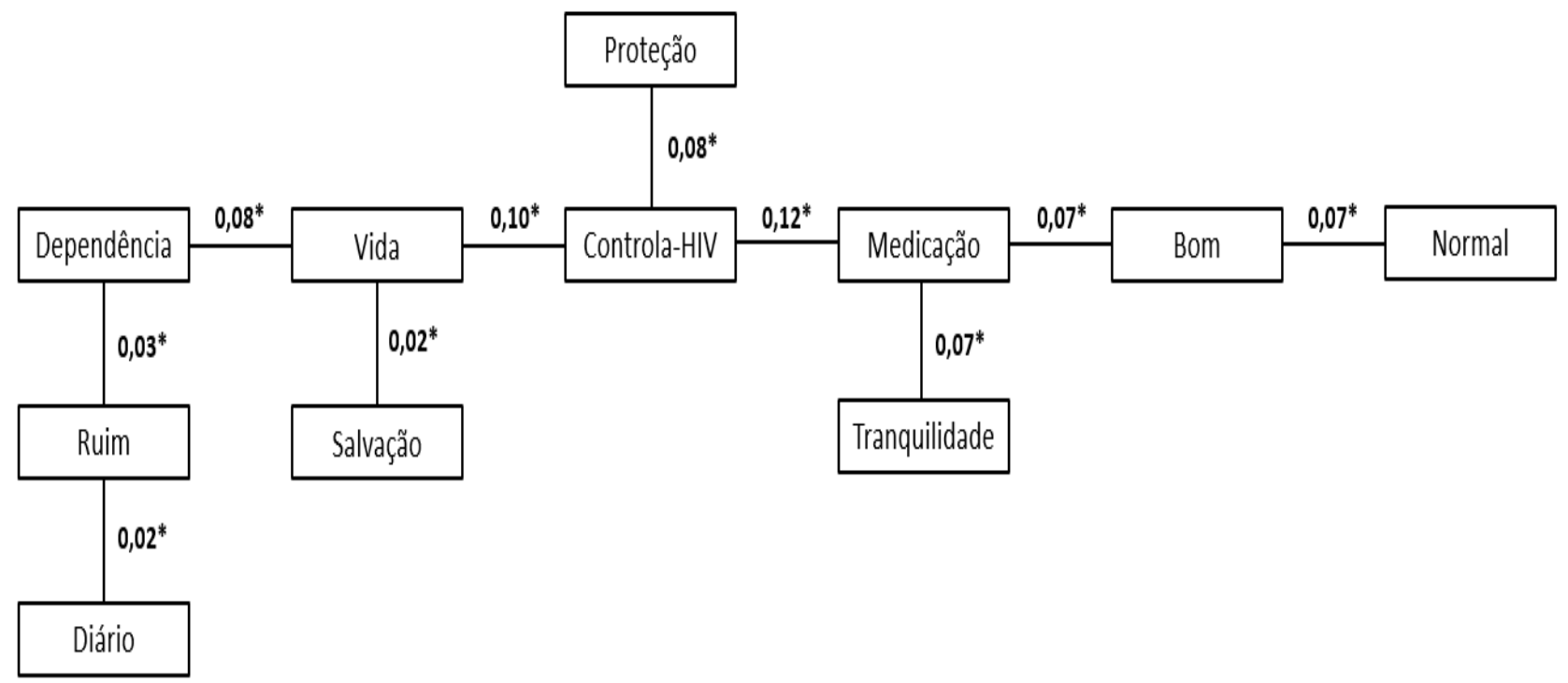

* Cada valor representa a força de ligação entre os termos evocados.

Figura 1 - Árvore máxima referente às evocações ao termo indutor antirretroviral, Rio de Janeiro, 2020

A Figura 1 evidencia que as palavras que estabeleceram maior número de conexões foram "vida”, com cinco ligações, “controla-HIV”, com quatro ligações e "medicamento” também com 4 ligações. A ligação com maior índice presente na árvore de similitude é a conexão entre as palavras 
Representações sociais da terapia antirretroviral para pessoas vivendo com HIV | 10 “controla-HIV” e “medicamento” $(0,12)$; seguida pela ligação entre “controla-HIV” e "vida” $(0,10)$. Estes dois eixos dão sequência às demais conexões entre os elementos que dão sentido as representações obtidas no estudo.

O termo que apresenta as ligações mais fortes da análise é "controla-HIV”, que se liga a “medicamento” $(0,12)$, “vida” $(0,10)$ e “proteção” $(0,08)$. Esses três elementos explicitam o significado fundamental da TARV, concebida como medicamento que confere proteção e vida. Cabe ressaltar as conexões dos elementos "dependência” e "vida” $(0,08)$, que sugerem a necessidade da TARV para continuar vivendo, ou seja, a dependência da TARV para manutenção da vida.

Destaca-se a possibilidade de centralidade do termo "controla-HIV", que realiza diferentes nexos com forças de ligação relevantes. Salienta-se as ligações de "controla-HIV” e "proteção" $(0,08)$; "vida" e dependência $(0,08)$; “medicamento" e "bom” $(0,07)$; "medicamento" e "tranquilidade" $(0,07)$; “dependência” e "ruim” $(0,03)$; “vida” e "salvação" $(0,02)$; “ruim” e "diário" $(0,02)$. Essas conexões, bem como as demais da TARV para o grupo estudado serão aprofundadas na discussão.

\section{Discussão}

As representações do antirretroviral apresentadas revelam as cognições dos participantes deste estudo, resultantes da experiência de utilização de uma terapia contínua para o tratamento de uma patologia crônica, incurável e altamente carregada de estigma. ${ }^{18}$ Observou-se um conjunto dos conteúdos representacionais que se destacaram para o grupo estudado.

O provável núcleo central contém termos que estruturam a representação do grupo, constituído por "controla-HIV", "bom" e "medicamento". O primeiro elemento remete ao que o grupo entende como sendo a finalidade do uso da TARV, e de acordo com alguns autores, pode retratar a familiaridade desses sujeitos por termos e noções mais técnico-científicas. ${ }^{19-20}$ Nesse sentido, o termo "bom" pode ter o papel de reforçar a favorável ação da TARV em conter a infecção. ${ }^{20} \mathrm{O}$ termo “medicamento" remete à objetivação da TARV em uma imagem afeita a algo físico que tem uma ação de tratamento e cura/controle de uma patologia. 
11 | Araújo SNO, Nascimento VC, Santos FK, Marques SC, Oliveira DC

A evocação "controla-HIV", expressa com a maior frequência absoluta, apresentou forte conexão com os termos "medicamento" e "vida", mostrando-se como o termo organizador da representação social. Essas ligações parecem indicar a finalidade principal da TARV, ou seja, a de controle do vírus, que, por sua vez, não está associado somente ao uso da medicação, mas também, à ideia de ser essencial para a manutenção da vida. Sendo assim, observa-se que, para o grupo estudado, a proteção da vida depende da utilização do medicamento.

A ligação entre "bom” e “medicamento" reforça a percepção mencionada, além de sugerir que para esse grupo o uso do medicamento se faz importante e necessário para que possam obter a qualidade e expectativa de vida tão boa quanto da população que não vive com HIV. ${ }^{18} \mathrm{~A}$ associação entre o uso da TARV e a proteção da vida é uma condição de tratamento alcançada mais recente, em função dos muitos avanços na terapêutica medicamentosa. Com o advento e evolução da TARV de alta eficácia, termos como autoaceitação e expectativas de vida, passaram a fazer parte do cotidiano de pessoas vivendo com HIV. Assim, ao longo do tempo houve uma remodelação da vida com HIV, antes mais fortemente associada à morte, e atualmente mais próxima à cronicidade da doença. Neste sentido, o tratamento vem sendo reconhecido como o meio para manter-se vivo. ${ }^{21}$

Essa percepção é reforçada pela presença da evocação de “vida”, única componente da primeira periferia, com uma conotação favorável a TARV. A importância da representação de vida para a TARV é relevante, ao considerar que o desejo de continuar vivendo é fundamental para a forte adesão à terapia. ${ }^{21}$ É importante destacar a necessidade do tratamento para fortalecer o sentimento de valorização da vida, do amor-próprio e do sentimento de esperança, fatores capazes de interferir na adesão ao tratamento. ${ }^{22-23}$ Para o grupo estudado, a ligação do termo "vida" com “salvação” tende a fortalecer o caráter positivo da imagem de “vida”, parecendo indicar que a TARV é reconhecida como meio para assegurar a sobrevivência.

A segunda periferia parece indicar uma ambivalência de sentimentos do grupo estudado: apesar do caráter positivo contido na imagem representada por tranquilidade, a indicação de dependência parece conter um aspecto indesejado da utilização do medicamento. Cabe mencionar a 
aparente tendência de o grupo referir-se à TARV como um elemento de normalidade. Isso fica evidente com os conteúdos representacionais presentes neste quadrante, bem como a partir das conexões entre "bom" e "normal” e tranquilidade" e "medicamento". Percebe-se uma associação entre a atitude positiva e um elemento que busca apontar para a normalidade da vida.

Esses achados reforçam uma tendência identificada por outro autor de "ilusão positiva", em que os sujeitos poderiam negar conscientemente a si mesmos, bem como a qualquer situação que possa influenciar de forma negativa o desejo de uma boa adesão ao tratamento e a resistência frente às dificuldades relacionadas. ${ }^{20}$ Cabe mencionar que o tratamento é caracterizado como um grande desafio, em face da complexidade do esquema terapêutico, das alterações da rotina e das reações adversas. ${ }^{7}$ Portanto, a expressão de uma normalidade atual pode se configurar como um movimento do grupo estudado no sentido de minimizar o eventual desgaste com os custos pessoais, entre eles, incluir uma medicação na rotina e reorganizar costumes para a utilização da terapia antirretroviral.

Contudo, a ligação entre os termos “dependência” e “ruim”, observados na análise de similitude, reforçam o significado negativo da TARV, provavelmente associado ao fato de a medicação ser indispensável para viver e da necessidade do seu uso diário, impactando a vida cotidianamente, além de se colocar como reafirmação da existência de uma doença que precisa ser enfrentada. Alguns indivíduos que usam a TARV sentem que os medicamentos constituem uma espécie de prisão a que estão submetidos, e que suas vidas estão ligadas aos horários, esquemas e dosagens exigidos pelos medicamentos. ${ }^{22}$

A zona de contraste comporta elementos com um caráter mais negativo, sobretudo quando relacionado a outros termos na análise de similitude que reforçam essa ideia, como dependência e diário, e esse fato pode indicar a possibilidade de existência de um subgrupo que representa o TARV de maneira diversa. Esses conceitos negativos sugerem diferentes experiências e tipos de enfrentamento individuais. ${ }^{20}$

Na segunda periferia, tem-se a evocação “dependência”, que é conteúdo que carrega uma atitude negativa, uma vez que sugere a subordinação ao TARV. A ligação das palavras "vida” e 
13 | Araújo SNO, Nascimento VC, Santos FK, Marques SC, Oliveira DC

“dependência” indica a obrigatoriedade do uso do tratamento para a manutenção da vida. ${ }^{20,22}$

Ainda na zona de contraste, o termo "diário", indica o sentido de rotina no uso da TARV, e, a ligação de "diário" e "ruim” na árvore de similitude, tende a apontá-la como algo negativo. Essa compreensão é reforçada, por estudos que apontam, os esquemas terapêuticos, como um aspecto gerador de dificuldade na atuação da TARV, aspecto negativo relacionado à adaptação à mudança de rotina diária, há uma relação da simplificação dos esquemas com uma adesão satisfatória. ${ }^{22,24}$

A representação dos quadrantes do núcleo central, primeira periferia e segunda periferia tendem a ter um posicionamento positivo sobre a TARV para esse grupo. Entretanto, a zona de contraste aponta atitudes negativas. Quanto às representações sociais da terapia antirretroviral, a partir dos dados do estudo, pode-se pensar em duas versões, a primeira relacionada a uma vida normal com uso regular das medicações. Já a segunda, refere-se ao antirretroviral como algo que gera dependência e sofrimento, mais característico de sujeitos com tratamento irregular/inadequado e baixa adesão. ${ }^{25}$

Entre os elementos apresentados pelo grupo, surgiram as evocações “ruim” e "bom”, e esses são termos que fazem uma avaliação oposta do TARV. Essa divergência pode ocorrer devido à complexidade da relação com o HIV, por este ser o causador de uma infecção incurável e estigmatizada com a existência de medicamentos que o podem controlar. ${ }^{20}$

Percebe-se que os conteúdos representacionais obtidos no quadro de quatro casas, expressam uma tendência mais positiva sobre a TARV, e esse resultado pode fazer parte de um processo de evolução das representações sociais do HIV. ${ }^{20,26}$ Essa evolução pode ocorrer quando elementos ligados à espiritualidade estão envolvidos no enfrentamento do TARV e ao diagnóstico. ${ }^{20}$

Nesse sentido, sabendo-se que as representações sociais permitem caracterizar os processos e relações nas quais grupos sociais estabelecem suas impressões acerca de dado objeto, a identificação dessas imagens pode auxiliar a compreensão do processo de adesão à terapia medicamentosa, bem como as ações dos profissionais da equipe de saúde. ${ }^{16,25}$ Entre os aspectos que impactam no recurso terapêutico, é relevante destacar que a equipe de saúde tem papel fundamental 
na aderência ao tratamento. ${ }^{27}$

Cabe ressaltar que os profissionais precisam estar cientes que a adesão compreende mais do que as questões farmacológicas, e envolve também alguns aspectos subjetivos e psicossociais do grupo, tais como: as variáveis individuais; as características do tratamento; as características da infecção pelo HIV/AIDS; a relação com o serviço de saúde e o apoio social. ${ }^{28}$

Portanto, é desejável que profissionais da saúde estejam capacitados de forma técnica e científica acerca do medicamento, além preparados para intervenções em questões individuais do paciente, visando um cuidado integral, promovendo a adesão e o autocuidado. ${ }^{11,29}$ Para tanto, o profissional deve ter conhecimento sobre a patologia e a terapia antirretroviral para estabelecer cuidados direcionados às pessoas vivendo com HIV, visando melhorar a sua expectativa de vida. ${ }^{23}$

O estudo identificou o termo "diário" e "dependência”, e ambas as evocações estão conectadas na análise de similitude ao termo "ruim", reforçando aspectos negativos do TARV. Pensando nisso, cabe ressaltar que o início do tratamento é um momento crítico e que deve ser construído em parceria com o indivíduo, dando subsídios para que o próprio possa tomar a atitude de aceitar o tratamento, de forma ativa e positiva. Fazendo-se, mais uma vez, necessária e importante as ações profissionais da saúde, no incentivo à aceitação da terapia, e sabendo que isso pode impactar diretamente na adesão aos medicamentos. ${ }^{22,25}$

Nesse contexto, a consulta de enfermagem é uma ferramenta importante para a criação da interação profissional-paciente e para um espaço de incentivo à adesão. Visto que, durante a consulta, além de compartilhar às questões mais subjetivas ao tratamento e ao diagnóstico, também é um ambiente favorável à orientação. A consulta de enfermagem é, ainda, um espaço para identificar eventuais dificuldades associadas ao início e ao uso regular da TARV, bem como, para traçar conjuntamente um plano de ação que torne possível minimizar o impacto desse evento. ${ }^{12}$

Cabe destacar que, à medida em que o contato entre a enfermagem e o paciente avança e se torna mais consistente, as dificuldades com a medicação e as dúvidas são minimizadas e esclarecidas, podendo promover maior adesão à terapia. As pessoas vivendo com HIV tendem a ter 
15 | Araújo SNO, Nascimento VC, Santos FK, Marques SC, Oliveira DC

na consulta de enfermagem um momento de suporte terapêutico. ${ }^{30}$

Dentre as limitações do estudo, observou-se dificuldades para realizar a coleta de dados no período planejado inicialmente, em face da suspensão dos serviços ambulatoriais por um período importante, pela pandemia da COVID-19.

\section{Conclusão}

Este estudo possibilitou perceber os diferentes conteúdos representacionais que a terapia antirretroviral traz para o grupo estudado, e identificar as relações de variada complexidade entre as imagens identificadas. A representação de uma medicação que controla o HIV, como um elemento bom para a consolidação de uma vida normal encontrou os aspectos indesejados que retratam a TARV como um elemento ruim pelo fato de gerar dependência e pelo uso diário.

Foi possível reconhecer, por meio dos dados, a estrutura representacional do TARV para o grupo estudado e, a partir dela, destacaram-se alguns aspectos de cunho positivo e negativo a serem observados para direcionar a atuação da equipe de enfermagem, o que possibilita uma abordagem mais específica. Espera-se, desta forma, contribuir para a promoção de uma melhor adesão ao esquema terapêutico, considerando a importância da TARV para assegurar a qualidade e a expectativa de vida neste segmento da população.

Portanto, a equipe de enfermagem tem uma inserção relevante neste processo, a partir da construção do vínculo terapêutico para orientação e incentivo diante de eventuais obstáculos encontrados pelos pacientes. Neste sentido, a consulta de enfermagem ganha especial importância, por trata-se de um espaço é possível dialogar, abordar os possíveis questionamentos e condições diversas capazes de interferir no uso da TARV.

\section{Referências}

1. UNAIDS Brasil. Estatísticas [Internet]. Brasília (DF): UNIAIDS Brasil; 2019 [acesso em 2019 out 20]. Disponível em: https://unaids.org.br/estatisticas

2. Ministério da Saúde (BR), Departamento de Doenças de Condições Crônicas e Infecções Sexualmente Transmissíveis. História da AIDS [Internet]. Brasília (DF): Ministério da Saúde; 2018 [acesso em 2019 set 10]. 
Disponível em: http://www.aids.gov.br/pt-br/centrais-de-conteudos/historia-aids-linha-do-tempo

3. Nunes Júnior SS, Ciosak SI. Terapia antirretroviral para HIV/AIDS: o estado da arte. Rev Enferm UFPE On Line. 2018;12(4):1103-11. doi: 10.5205/1981-8963-v12i4a231267p1103-11011-2018

4. Menezes EG, Santos SRF, Melo GZS, Torrente G, Pinto AS, Goiabeira YNLA. Fatores associados à não adesão dos antirretrovirais em portadores de HIV/AIDS. Acta Paul Enferm. 2018;31(3):299-304. doi: 10.1590/1982-0194201800042

5. Barros SG, Vieira-da-Silva LM. A terapia antirretroviral combinada, a política de controle da AIDS e as transformações do Espaço AIDS no Brasil dos anos 1990. Saúde Debate. 2017;41(3 N Esp):114-28. doi: 10.1590/0103-11042017s309

6. Freitas JP, Sousa LRM, Cruz MCMA, Caldeira NMVP, Gir E. Terapia com antirretrovirais: grau de adesão e a percepção dos indivíduos com HIV/AIDS. Acta Paul Enferm. 2018;31(3):327-33. doi: 10.1590/19820194201800046

7. Ministério da Saúde (BR), Departamento de Vigilância, Prevenção e Controle das Infecções Sexualmente Transmissíveis. Protocolo clínico e diretrizes terapêuticas para manejo da infecção pelo HIV em adultos [Internet]. Brasília (DF): Ministério da Saúde; 2018 [acesso em 2019 ago 28]. Disponível em: http://www.aids.gov.br/pt-br/pub/2013/protocolo-clinico-e-diretrizes-terapeuticas-para-manejo-da-infeccaopelo-hiv-em-adultos

8. Sá BTP, Martins MR, Boger B, Dias FA, Rattmann YD. Estimativa de adesão ao medicamento antirretroviral coformulado 3 em 1. ABCS Health Sci. 2018;43(3):136-40. doi: 10.7322/abcshs.v43i3.1002

9. Silva CL, Cubas MR, Silva LLX, Cabral LPA, Grden CRB, Nichiata LYI. Diagnósticos de enfermagem associados às necessidades humanas no enfrentamento do HIV. Acta Paul Enferm. 2019;32(1):18-26. doi: 10.1590/1982-0194201900004

10. Freitas MIF, Bonolo PF, Miranda WD, Guimarães MDC. Interações sociais e a adesão à terapia antiretroviral de pessoas vivendo com HIV/AIDS. REME Rev Min Enferm. 2017;21:e-1001. doi: 10.5935/14152762.20170011

11. Moraes DCA, Oliveira RC, Prado AVA, Cabral JR, Corrêa CA, Albuquerque MMB. O conhecimento de pessoas vivendo com HIV/AIDS sobre a Terapia Antirretroviral. Enfermería Glob. 2018;49:111-26. doi: 10.6018/eglobal.17.1.274001

12. Souza LRM, Moura LKB, Valle ARMC, Magalhães RLB, Moura MEB. Representações sociais do HIV/Aids por idosos e a interface com a prevenção. Rev Bras Enferm. 2019;72(5):1192-9. doi: 10.1590/00347167-2017-0748

13. Chamon EMQO, Lacerda PG, Marcondes NAV. Um breve revisar de literatura sobre a teoria das representações sociais. Rev Ensino Educ Ciênc Hum; 2017;18(4):451-7. doi: 10.17921/24478733.2017v18n4p451-457

14. Jodelet D. As representações sociais. Rio de Janeiro (RJ): EdUERJ; 2001. 420 p.

15. Paula FN, Oliveira JHC, Santos SRM. Representações sociais e sociedades. LexCult. 2018;2(1): 10-27. doi: 10.30749/2594-8261.v2n1p10-27 
16. Wolter R. Abordagem estrutural das representações sociais: pontes entre teoria e método. Psico-USF. 2018;23(4):621-31. doi: 10.1590/1413-82712018230403

17. Moura SRB, Marques Júnior MASS, Rocha AKL, Vieira JPPN, Mesquita GV, Brito JNPO. Análise de similitude dos fatores associados à queda de idosos. Rev Interdiscip. 2015;8(1):167-73.

18. Bezerra EO, Pereira MLD, Maranhão TA, Monteiro PV, Brito GCB, Chaves ACP. Análise estrutural das representações sociais sobre AIDS entre pessoas que vivem com vírus da imunodeficiência humana. Texto Contexto Enferm. 2018;27(2):e6200015. doi: 10.1590/0104-070720180006200015

19. Torres TL, Camargo BV. Representações sociais da Aids e da terapia anti-retroviral para pessoas vivendo com HIV. Psicol Teor e Pesqui [Internet]. 2008 [acesso em 2020 nov 15];10(1):64-78. Disponível em: http://pepsic.bvsalud.org/pdf/ptp/v10n1/v10n1a06.pdf

20. Costa TL, Oliveira DC, Formozo GA, Gomes AMT. Análise estrutural das representações sociais da terapia antirretroviral entre pessoas que vivem com HIV/Aids: possibilidades de convivência, normatividade e ressignificação. Psicol Saber Soc. 2013;2(1):104-14. doi: 10.12957/psi.saber.soc.2013.6931

21. Gomes MP, Barbosa DJ, Gomes AMT, Silva GA, Souza FBA, Silva ALB. A fé e a esperança como ferramenta de suporte frente ao diagnóstico positivo de HIV. Fragm Cult. 2020;30(1):117-23. doi: 10.18224/frag.v30i1.7663

22. Teixeira MG, Silva GA. A representação do portador do vírus da imunodeficiência humana sobre o tratamento com os anti-retrovirais. Rev Esc Enferm USP. 2008;42(4):729-36. doi: 10.1590/S008062342008000400016

23. Figueiredo MSBR, Patrício ACF, Silva DF, Leite MAP, Santos JS, Rodrigues BFL, et al. Sofrimento mental, desesperança e adesão a terapia antirretroviral de pessoas com HIV/AIDS. REME Rev Min Enferm. 2020;24:e-1338. doi: 10.5935/1415-276220200075

24. Carvalho PP, Barroso SM, Coelho HC, Penaforte FRO. Fatores associados à adesão à terapia antirretroviral em adultos: revisão integrativa de literatura. Ciênc Saúde Colet. 2019;24(7):2543-55. doi: 10.1590/1413-81232018247.22312017

25. Camargo BV, Bousfield ABS, Giacomozzi AI, Koelzer LP. Representações sociais e adesão ao tratamento antirretroviral. Liberabit [Internet]. 2014 [acesso em 2020 nov 02];20(2):229-38. Disponível em: http://www.scielo.org.pe/pdf/liber/v20n2/a04v20n2.pdf

26. Suto CSS, Marques SC, Oliveira DC, Oliveira JF, Paiva MS. Profissionais de saúde falam mais sobre cuidado e menos sobre síndrome da imunodeficiência adquirida. Cogitare Enferm. 2017;(22)3:e49981. doi: 10.5380/ce.v22i3.49981

27. Silva RAR, Nelson ARC, Duarte FHS, Prado NCC, Holanda JRR, Costa DARS. Avaliação da adesão à terapia antirretroviral em pacientes com AIDS. Rev Pesq Cuid Fundam. 2017;9(1):15-20. doi: 10.9789/21755361.2017

28. Carvalho PP, Barroso SM, Coelho HC, Penaforte FRO. Fatores associados à adesão à Terapia Antirretroviral em adultos: revisão integrativa de literatura. Ciênc Saúde Colet. 2019;24(7):2543-55. doi: 10.1590/1413-81232018247.22312017

29. Silva RAR, Costa MMN, Souza Neto VL, Silva BCO, Costa CS, Andrade IFC. Falta de adesão em pessoas 
vivendo com HIV: acurácia das características definidoras do diagnóstico de enfermagem. Rev Latinoam Enferm. 2017;25:e2940. doi: 10.1590/1518-8345.1582.2940

30. Isoldil DMR, Carvalho FPB, Simpson CA. Análise contextual da assistência de enfermagem à pessoa com HIV/Aids. Rev Pesq Cuid Fundam. 2017;9(1):273-8. doi: 10.9789/2175-5361.2017.v9i1.273-278

Editora Científica Chefe: Cristiane Cardoso de Paula

Editora associada: Nara Marilene Oliveira Girardon-Perlini

\section{Autor correspondente}

Shaiene Naiara Oliveira de Araújo

E-mail: shaienenoa@gmail.com

Endereço: Rua Godofredo Silva, 164, Vila da Penha, Rio de Janeiro, RJ, Brasil. CEP:21221020

\section{Contribuições de Autoria}

\section{1 - Shaiene Naiara Oliveira de Araújo}

Concepção ou desenho do estudo/pesquisa, análise e/ou interpretação dos dados, revisão final com participação crítica e intelectual no manuscrito.

\section{2 - Vivian Corrêa Nascimento}

Concepção ou desenho do estudo/pesquisa, análise e/ou interpretação dos dados, revisão final com participação crítica e intelectual no manuscrito.

\section{3 - Felipe Kaezer dos Santos}

Concepção ou desenho do estudo/pesquisa, análise e/ou interpretação dos dados, revisão final com participação crítica e intelectual no manuscrito.

\section{4 - Sergio Corrêa Marques}

Revisão final com participação crítica e intelectual no manuscrito.

\section{5 - Denize Cristina Oliveira}

Revisão final com participação crítica e intelectual no manuscrito.

\section{Como citar este artigo}

Araújo SNO, Nascimento VC, Santos FK, Marques SC, Oliveira DC. Social representations of antiretroviral therapy for people living with HIV. Rev. Enferm. UFSM. 2021 [Access in: Years Month Day]; vol.11 e54: 1-18. DOI: https://doi.org/10.5902/2179769263378 